
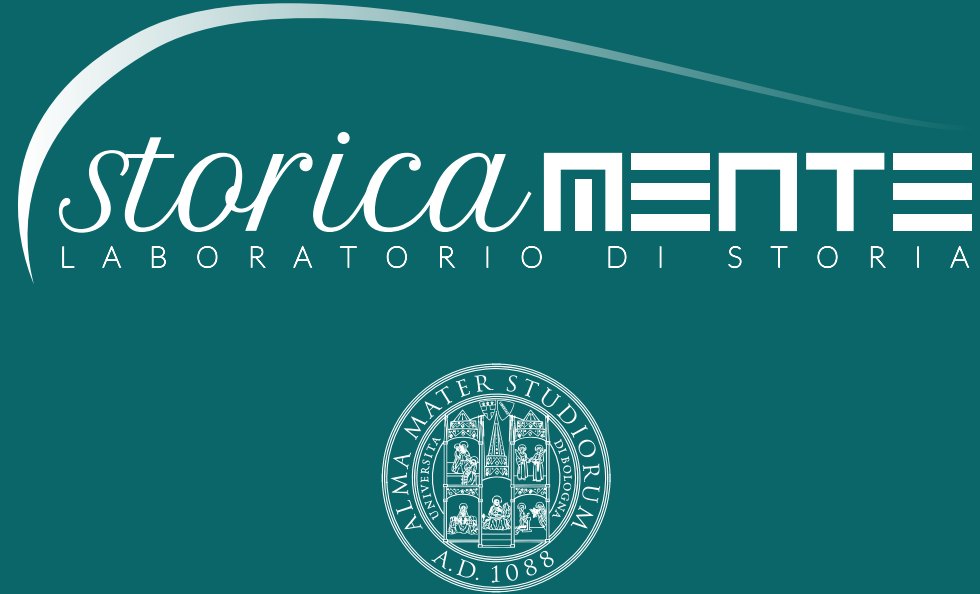

ALMA MATER STUDIORUM

Università di Bologna

Dipartimento di Storia Culture Civiltà

COMUNICARE STORIA

LA STORIA A SCUOLA OGGI.

INSEGNARE STORIA

NELLA SCUOLA PRIMARIA 


\section{STORICAMENTE.ORG}

\section{Laboratorio di Storia}

Luigi Cajani

I recenti programmi di storia per la scuola italiana

Numero 15 - 2019

ISSN: $1825-411 \mathrm{X}$

Art. 52

pp. 1-42

DOI: $10.12977 /$ stor798

Editore: BraDypUS

Data di pubblicazione: 10/10/2020

Sezione: Comunicare storia: "La Storia a scuola oggi. Insegnare storia nella scuola primaria” 


\title{
I recenti programmi di storia per la scuola italiana
}

\author{
LUIGI CAJANI
}

Univ. Sapienza, Dipartimento di Storia Antropologia

Religioni Arte Spettacolo

This article examines the history programmes for Italian schools of all orders and levels over a period of more than ten years, culminating in 2012, during which several reforms were carried out. In particular the article focuses on Eurocentrism, on world history and on cultural identity, as well as on the political, cultural and historiographical contexts that determined the development of the different programmes.

\section{Presentazione}

Ripropongo qui integralmente un articolo del 2014 sulle vicende recenti dei programmi di storia per la scuola italiana, su richiesta dei curatori di questo numero e grazie alla cortesia della redazione del Laboratorio dell'ISPF, che lo pubblicò allora1. In questi ultimi sei anni non ci sono state novità per quanto riguarda questi programmi, ma vanno comunque ricordati tre episodi che han* Si ringrazia l'Istituto per la storia del pensiero filosofico e scientifico moderno (ISPFCNR) per aver concesso la pubblicazione dell'articolo apparso su Laboratorio del ISPF (XI, 2014) e qui riproposto con un aggiornamento dell'Autore e l'apparato delle fonti legislative citate.

Cajani, Luigi. I recenti programmi di storia per la scuola italiana. Laboratorio dell'ISPF. 2014, vol. XI. DOI: 10.12862/ispf14L205 
no in vario modo toccato l'insegnamento della storia: la riduzione del numero di ore negli istituti professionali, l'eliminazione di una prova scritta di storia alla maturità e il decreto sull'educazione civica.

Il primo episodio è legato a due decreti dell'aprile 2017 e del luglio 20182, emanati in attuazione delle Legge delega nota come Buona Scuola3. In base ad essi è stato creato nel biennio degli Istituti professionali un asse storicosociale, comprendente storia, diritto ed economia e geografia. Quest'ultima disciplina era assente dal programma precedente 4 , e poiché il monte ore dell'asse è rimasto di quattro ore, tante quante erano precedentemente assegnate a storia e diritto ed economia, il suo inserimento ha portato a un dimezzamento delle ore assegnate alla storia, cioè da due a una a settimana.

Il secondo episodio risale all'ottobre del 2018, quando il ministro Marco Bussetti rese noti i risultati Commissione sulla riforma degli esami di Stato, presieduta da Luca Serianni, nella quale, fra i tanti provvedimenti, c'era l'eliminazione di una delle due prove di storia, quella denominata "tipologia C", con l'argomentazione che ormai era scelta solo dall'1\% degli studenti. L'iniziativa

Decreto Legislativo 13 aprile 2017, n. 61: Revisione dei percorsi dell'istruzione professionale nel rispetto dell'articolo 117 della Costituzione, nonché raccordo con i percorsi dell'istruzione e formazione professionale, a norma dell'articolo 1, commi 180 e 181, lettera d), della legge 13 luglio 2015, n. 107, in "Gazzetta Ufficiale della Repubblica Italiana", Serie generale, n. 112, 16 maggio 2017; Ministero dell'Istruzione, dell'Università e della Ricerca, Decreto 24 maggio 2018, n. 92: Regolamento recante la disciplina dei profili di uscita degli indirizzi di studio dei percorsi di istruzione professionale, ai sensi dell'articolo 3, comma 3, del decreto legislativo 13 aprile 2017, n. 61, recante la revisione dei percorsi dell'istruzione professionale nel rispetto dell'articolo 117 della Costituzione, nonché raccordo con i percorsi dell'istruzione e formazione professionale, a norma dell'articolo 1, commi 180 e 181, lettera d), della legge 13 luglio 2015, n. 107,in "Gazzetta Ufficiale della Repubblica Italiana”, Serie generale, n. 173, 27 luglio 2018, Supplemento ordinario n. 35/L.

3 Legge 13 luglio 2015, n. 107: Riforma del sistema nazionale di istruzione e formazione e delega per il riordino delle disposizioni legislative vigenti, in "Gazzetta Ufficiale della Repubblica Italiana”, Serie generale, n. 152, 15 luglio 2015.

4 Decreto del Presidente della Repubblica 15 marzo 2010, n. 87:Regolamento recante norme per il riordino degli istituti professionali, a norma dell'articolo 64, comma 4, del decreto-legge 25 giugno 2008, n. 112, convertito, con modificazioni, dalla legge 6 agosto 2008, n. 133, in "Gazzetta Ufficiale della Repubblica Italiana”, Serie generale, n. 137, 15 giugno 2010, Supplemento ordinario n. 128. 
suscitò un gran numero di reazioni negative, a partire dalle associazioni degli storici italiani5, e nel dibattito emerse anche un riflessione importante sul perché questa prova aveva così scarso successo, e cioè il fatto che le tracce erano riferite quasi esclusivamente agli eventi più recenti, che di regola non venivano insegnati. A questo impegno in difesa della storia si è collega l'appello "La storia è un bene comune”, pubblicato alcuni mesi dopo, nell'aprile del 2019, firmato da Andrea Giardina, presidente della Giunta centrale per gli studi storici, dallo scrittore Andrea Camilleri e dalla senatrice Liliana Segre e promosso dal quotidiano "la Repubblica"6. In questo testo, che venne largamente sottoscritto nel mondo accademico e intellettuale, si affermava che la conoscen$z a$ della storia "è un principio di democrazia e uguaglianza fra i cittadini", in quanto esprime "un sapere critico non uniforme, non omogeneo, che rifuta in conformismo e vive nel dialogo". E in conclusione si chiedeva il ripristino della prova di storia all'esame di maturità, l'aumento e non la diminuzione delle ore di insegnamento nelle scuole (con un esplicito riferimento agli Istituti professionali), e infine il sostegno alla ricerca universitaria, con l'ampliamento dell'accesso ai giovani studiosi. Le proteste ebbero un parziale successo, perché alla fine la prova di storia venne reinserita nell'esame di maturità del 20197. La questione delle ore di insegnamento si ripresenta però nel terzo episodio, cioè la legge sull'insegnamento dell'educazione civica, approvata nell'agosto del 20198. Con essa l'educazione civica, che ha sempre avuto una storia travagliata nella scuola italiana, viene introdotta in ogni ciclo come disciplina

Addio al tema di storia alla Maturità, la protesta degli storici: "Che errore», in "Corriere della Sera", 9 ottobre 2018. Si veda anche: Valentina Santarpia, "Che errore abolire la Storia alla Maturità». Lrappello degli esperti, in "Corriere della Sera", 10 ottobre 2018; Ilaria Venturi, Nessuno tocchi la nostra maestra storia, in "la Repubblica", 10 ottobre 2018 .

6 Il manifesto. Il passato è un bene comune, in "la Repubblica", 126 aprile 2019.

7 https://www.istruzione.it/esame_di_stato/201819/Italiano/Ordinaria/P000_ ORD19.pdf

8 Legge 20 agosto 2019 n. 92: Introduzione dell'insegnamento scolastico dell'educazione civica, in "Gazzetta Ufficiale della Repubblica Italiana”, Serie generale, n. 195, 21 agosto 2019. 
autonoma, con tanto di valutazione finale, e con non meno di 33 ore annue, da ricavare all'interno del monte ore complessivo attuale, giacché si tratta anche in questo caso di una riforma a costo zero in termini di orario. L'educazione civica in questa versione italiana è una disciplina dichiaratamente trasversale, i cui molti temi, raggruppati sotto Costituzione, sviluppo sostenibile e cittadinanza digitale, coinvolgono non solo la storia, che nella cultura scolastica italiana è da sempre il veicolo privilegiato dell'educazione civica, ma anche le scienze naturali, la geografia, la letteratura e il diritto e l'economia9. Tutte discipline a cui verranno quindi sottratte ore destinate allo svolgimento dei rispettivi programmi, in misura peraltro variabile a seconda delle scuole, giacché è loro demandata la revisione in autonomia dei curricoli di istituto per adeguarli alle nuove disposizioni. In ogni caso, l'auspicio di un aumento delle ore di insegnamento della storia, espresso nell'appello, è per ora frustrato.

\section{Berlinguer e il Novecento}

La riforma generale della scuola italiana, la prima dalla fondazione della Repubblica, ha avuto un percorso molto lungo e accidentato, durato oltre dieci anni, perché travagliato dalle alterne vicende politiche che hanno investito sia la struttura del sistema scolastico che i programmi d'insegnamento. Fra tutti, i programmi di storia sono stati senza paragone quelli maggiormente colpiti dalle polemiche, il che non sorprende visto che questa materia scolastica è sempre quella politicamente più sensibile: ma queste polemiche hanno gettato anche un'interessante luce sulla storiografia italiana.

Polemiche sull'insegnamento della storia iniziarono in verità già prima che il quadro della nuova scuola venisse delineato, quando nel 1996 il

\footnotetext{
Ministero dell'Istruzione, Decreto Ministeriale n.35 del 22 giugno 2020: Linee guida per l'insegnamento dell'educazione civica, ai sensi dell'articolo 3 della legge 20 agosto 2019, n. 92, Allegato A.
} 
ministro della Pubblica Istruzione Luigi Berlinguer stabilì che nell'ultimo anno della scuola media e della scuola secondaria superiore si insegnasse solo la storia del Novecento ${ }^{10}$, e non anche quella dell'Ottocento, come era tradizione dell'Italia repubblicana. Questa scelta era già stata praticata da quelle scuole superiori, che dall'inizio degli anni Novanta avevano adottato in via sperimentale i programmi della Commissione Brocca (Piani di studio 1992, 139-42). Berlinguer nella premessa del decreto motivava questa innovazione con «l'esigenza pedagogico-culturale di dedicare un più ampio spazio alla trattazione di avvenimenti recenti di notevole rilievo storico-politico», un'esigenza che non era possibile soddisfare con la precedente ripartizione cronologica dei programmi. Va notato che in molti altri stati europei, come la Francia e la Germania, la storia del Novecento ha sempre avuto uno spazio maggiore di quello che aveva in Italia, e che quindi l'Italia costituiva piuttosto un'eccezione. Ma l'insegnamento della storia contemporanea è stato una questione spinosa sin dai primi anni della Repubblica, oggetto di polemiche di carattere soprattutto politico, che hanno visto la contrapposizione di destra e sinistra (Cajani 2003). Contrapposizione che riesplose anche in questo caso in un vasto dibattito. Fra i fautori" Vittorio Foa sottolineava l'importanza di uno studio attento del Novecento nella formazione dei giovani: «Gli studenti devono studiare questo secolo per capire il rapporto fra il ricordo del passato e il futuro

\footnotetext{
10 Si veda il decreto del ministro della Pubblica Istruzione n. 682 del 4.11.1996: Modifiche delle disposizioni relative alla suddivisione annuale del programma di Storia. Il decreto riguardava anche la scuola elementare: ai docenti del secondo ciclo si raccomandava di introdurre «la conoscenza dei più importanti eventi dell'ultimo secolo, tenendo presenti le capacità e i modi di apprendimento propri degli alunni e l'esigenza di un continuo riferimento alla concreta realtà in cui essi sono inseriti».

11 Tortello, M. 1996. "Berlinguer rivoluziona l'ora di storia." La Stampa, 1 ottobre; Ruggeri, C. 1996. "Studiamo la storia di oggi." Il Corriere della Sera, 1 ottobre; De Luna, G. 1996. "La scuola tra storia e musica." L’Unità, 4 novembre; Galasso, G. 1996. "La materia c'è, attendiamo lo svolgimento." Il Mattino, 5 novembre.
} 
della loro vita»'2. Fra gli oppositori, alcuni muovevano critiche sul piano didattico e culturale, lamentando il sacrificio dello studio dell'antichità greca e romana ${ }^{13}$, del medioevo ${ }^{14} \mathrm{o}$ del Risorgimento, a causa della compressione del programma negli anni precedenti all'ultimo, soprattutto nella scuola media ${ }^{15}$; altri si muovevano sul piano politico, riprendendo l'annosa polemica della destra italiana sulla strumentalizzazione politica dell'insegnamento della storia contemporanea. Uno dei più autorevoli giornalisti conservatori italiani, Indro Montanelli, scriveva: «Esistono dei testi scolastici di Storia contemporanea dei quali ci si possa fidare come equilibrio e imparzialità? Non ne conosco» ${ }^{16}$. Un altro giornalista di destra, Marcello Veneziani, in una risposta a Berlinguer, attaccava anch'egli i libri di testo, lamentando che ignorassero gli esponenti del cosiddetto revisionismo storico:

Veda i libri di testo nelle scuole. Viaggiano con un trentennio di ritardo, si sente ancora odore di Unione Sovietica e di Cina maoista. E non si avverte traccia di quello che è stato definito, a torto o a ragione, il revisionismo storico degli ultimi trent'anni: parlo di De Felice e Romeo, di Furet e di Sternhell, di Nolte e Hillgruber....c’è il rischio che la sua buona idea di far studiare il Novecento produca un cattivo effetto: il ritorno della storia ideologica e manichea ${ }^{17}$.

12 Si veda l'intervista di Mario Ajello: Ajello, M. 1996. "Cari ragazzi, finalmente scoprirete un secolo. Il vostro." Il Messaggero, 1 ottobre.

13 Particolarmente attivo in questa direzione è stato Fabrizio Polacco, che fondò l'associazione Prisma (Progetto per la rivalutazione dell'insegnamento e dello studio del mondo antico) e redasse una proposta di modifica al decreto Berlinguer (Polacco 1999, 141-43).

14 Frugoni, C. 1996. "Ma così si uccide il Medioevo." La Repubblica, 9 novembre.

15 Nucci, A. 1996. "Tutti i difetti del decreto Berlinguer." Il Resto del Carlino, 12 novembre; Casalegno, A. 1996. "Ignorare il passato non aiuta a capire il nostro presente." Il Sole 24 ore, 16 novembre; Maffioli, F. 1996. "Critiche anche dall'«Osservatore»." Il Giornale, 24 novembre.

${ }_{16}$ "Il progetto Berlinguer? Che brividi! (La stanza di Montanelli)." Il Corriere della Sera, 5 ottobre 1996.

17 "Novecento." Epoca, 18 ottobre 1996. 
Molte altre voci peraltro negavano che esistesse un pericolo simile. Lo storico Giorgio Spini, autore di un importante e longevo manuale di storia per le scuole superiori, osservava che il timore di una strumentalizzazione politica della storia recente era ormai inattuale:

oggi non c'è più questo problema, con la rivoluzione dell'89 la guerra è finita, l'ha vinta l'America, non c'è più la guerra fredda, non c'è più il pericolo che la lezione si trasformi in propaganda. Quei fantasmi si vanno dileguando ${ }^{18}$.

Un altro storico, Francesco Renda, metteva invece in discussione proprio l'esistenza del problema della faziosità, con un'importante messa a punto della questione:

Non bisogna preoccuparsi della faziosità, che può essere utile perché significa passione per la materia, ma della menzogna. Questa materia è ricerca della verità, il problema sta nella falsificazione dei fatti e non nel modo in cui ciascuno inevitabilmente legge un avvenimento in base alle proprie convinzioni... Il fascista insegnerà da fascista, il pidiessino da tale. Non c'è da temere, perché siamo in tempo di democrazia e di pluralismo conoscitivo. Bisogna avere paura della menzogna, perché la verità faziosa è utile purché ci sia questo bisogno di verità. La storia senza passione non ha senso. Il problema sta nella ricerca dei fatti senza falsificazioni, che deve stare alla base di ogni insegnamento storico ${ }^{19}$.

Nonostante le critiche, comunque, Berlinguer mantenne il decreto, e passò all'impostazione della riforma del sistema scolastico italiano dapprima con la nomina di una "Commissione dei Saggi" (Le conoscenze 1997), incaricata di definirne i contorni culturali generali, e poi promulgando la legge 30/200020, che definì l'architettura della nuova scuo-

18 Si veda l'intervista di Marina Cavalleri: Cavalleri, M. 1996. "Quale novità? Nei programmi c'è sempre stato." La Repubblica, 1 ottobre.

19 Si veda l'intervista di Gerardo Marrone: Marrone, G. 1996. "Diamo spazio anche alle vicende siciliane." Giornale di Sicilia, 1 ottobre.

20 Legge 10 febbraio 2000, n. 30: "Legge-quadro in materia di riordino dei cicli dell'istruzione." Gazzetta ufficiale 44, 23 febbraio 2000. 
la italiana. Le innovazioni fondamentali contenute in questa legge riguardavano la durata complessiva del percorso scolastico, ridotto da 13 a 12 anni, la durata dell'obbligo scolastico, aumentato da 8 a 9 anni, e la divisione non più in tre cicli ma in due $(7$ anni di scuola di base +5 di scuola superiore), sicché la scansione fra il primo e il secondo intersecava il percorso dell'obbligo scolastico. Pertanto, il biennio iniziale della scuola superiore, con cui appunto si concludeva l'obbligo, assumeva il carattere di snodo e di transizione fra la scuola di base, uguale per tutti, e il triennio conclusivo della scuola superiore, nettamente distinto nei vari ordini. Questa configurazione del biennio si poneva in continuità con una lunga riflessione sulla riforma della scuola italiana, nella quale l'elevazione dell'obbligo scolastico andava di pari passo con una separazione progressiva e non più immediata fra i vari indirizzi, attraverso l'individuazione di una base di conoscenze comuni a tutti, mentre la specializzazione e la separazione dei vari indirizzi sarebbe stata realizzata nel triennio successivo. Nel 1985 il ministro della Pubblica Istruzione Franca Falcucci aveva presentato un progetto di riforma, rimasto inattuato, in cui si prevedeva che nel biennio ci fosse una consistente area comune di materie, pari a 26 ore settimanali su 36ㄹ. Successivamente, la Commissione Brocca aveva mantenuto la stessa struttura in due aree, quella delle materie comuni, per complessive 22 o 23 ore settimanali ${ }^{22}$, e quella delle materie specifiche, il cui carico orario variava da indirizzo a indirizzo.

Le materie comuni erano: italiano, lingua straniera, storia, educazione civica, giuridica, economica (che comprendeva la geografia economica), matematica-informatica, fisica, biologia, chimica, scienze della terra, educazione fisica, religione (Il biennio 1986, 171-248). Su questo programma e sul dibattito che l'ha circondato si veda Di Pietro 1991, 137-53.

${ }_{22}$ Le materie comuni erano: educazione fisica, religione, italiano, lingua straniera 1, storia, diritto ed economia, matematica e informatica, scienze della terra (al primo anno), biologia (al secondo anno) (Piani di studio 1991, 23, 28-29). 


\section{La riforma De Mauro}

Con il cambio di governo, sempre di centrosinistra, il compito di realizzare la riforma della scuola passò nelle mani di Tullio De Mauro, che successe a Berlinguer il 25 aprile del 2000. De Mauro nominò una commissione composta da circa 250 esperti, che nella prima fase dei suoi lavori, durante l'estate del 2000, definì l'articolazione dei vari cicli. Particolare attenzione venne riservata al biennio, l'elemento più delicato del nuovo sistema, per il quale venne ripresa l'articolazione in due aree disciplinari: un'area comune, costituita dalle discipline che dovevano rappresentare la base culturale di tutti i futuri cittadini, e un'area di indirizzo, costituita appunto dalle discipline che caratterizzavano gli indirizzi stessi e avviavano le specializzazioni del triennio successivo ${ }^{23}$. La definizione di quali sarebbero state le materie comuni venne rimandata alla fase successiva dei lavori della commissione, ma a mo' d'esempio vennero fatte due ipotesi di articolazione per scienze e storia. Per la storia si propose una prima scansione all'interno della scuola di base e una seconda alla fine del biennio della scuola superiore:

Per la storia si prospettano tre fasi: a) la prima attenta alla "grammatica» della disciplina e cioè agli strumenti che ne permettono la conoscenza; b) la seconda alla «sistematica» cioè alla conoscenza organica della materia; c) la terza ai «percorsi» che consentono approfondimenti particolari. Le tre fasi coprono l'intero percorso di istruzione (la fine dell'obbligo coinciderebbe con la conclusione della fase $\mathrm{b}$ - conoscenza sistematica $)^{24}$.

La seconda fase dei lavori, che prese il via nel gennaio 2001, venne dedicata alla scuola di base. La commissione venne suddivisa in vari

\footnotetext{
"Scuola secondaria del Sottogruppo di lavoro n. 7c, Coordinatore: prof. Mario Ambel, Moderatore: Isp. Chiara Croce." Annali della Pubblica Istruzione 46 (3-4), 2000, 233.

24 "Scuola di base del Sottogruppo di lavoro n. 7b, Coordinatore: isp. Alberto Alberti, Moderatore: Isp. Carlo Petracca." Annali della Pubblica Istruzione 46 (3-4), 2000, $220-1$.
} 
sottogruppi di lavoro a seconda delle discipline, e uno di questi si occupò dell'insieme formato da storia, geografia e scienze sociali. Come già avveniva nei programmi per la scuola elementare del $1985^{25}$, (allora però si parlava di studi sociali), queste tre materie erano appunto collegate fra di loro, ma in questo caso se ne previde una progressiva autonomizzazione in vista del biennio. Si decise inoltre che la storia sarebbe dovuta essere senz'altro una delle materie comuni del biennio, e quindi se ne disegnò un programma (anzi: "curricolo", secondo la nuova definizione ministeriale) che continuava anche nel biennio. La decisione sulle materie comuni del biennio, a dire il vero, sarebbe stata compito della fase successiva dei lavori, quando la Commissione si sarebbe occupata della scuola secondaria, ma per quanto riguardava la storia l'impostazione cronologica del curricolo, che si sarebbe appunto concluso alla fine del biennio, rendeva necessario decidere in anticipo. I curricoli di geografia e scienze sociali, invece, non avevano costrizioni paragonabili alla continuità cronologica, e la parte relativa al biennio poteva essere rimandata senza problemi. Il sottogruppo riprese poi l'indicazione della prima fase dei lavori per quanto riguardava la scansione tripartita del curricolo di storia. Un'assoluta e fondamentale novità sul piano dei contenuti fu infine l'accoglimento quasi unanime della proposta, fatta dalla pattuglia di storici presenti, di sostituire la tradizionale impostazione eurocentrica con un'impostazione mondiale, ispirandosi in questo ai più recenti sviluppi della ricerca internazionale, che fra l'altro proprio pochi anni prima avevano ispirato la riforma dell'insegnamento della storia negli USA ${ }^{26}$.

La fase di "grammatica" del programma di storia venne assegnata al terzo e al quarto anno della scuola di base, in cui era previsto che le tre

Decreto del presidente della Repubblica 12 febbraio 1985, 104: "Approvazione dei nuovi programmi didattici per la scuola primaria.” Gazzetta Ufficiale 76, 29 marzo 1985, n. 76, Supplemento ordinario, con le correzioni disposte con avviso di rettifica pubblicato in Gazzetta Ufficiale 105, 6 maggio 1985.

26 National Standards 1996; Lopes Don 2003; Nash, Crabtree e Dunn 1999. 
materie, pur iniziando ad assumere i primi caratteri autonomi, restassero fortemente intrecciate fra di loro intorno a un oggetto comune, $i$ "quadri di società": società di cacciatori e raccoglitori, società agricole, società pastorali nomadiche, società urbane, società industriali e postindustriali. Questi quadri di società richiamavano i quadri di civiltà introdotti nei programmi per la scuola elementare del 1985, ma si era scelto in termine "società" per fare riferimento a contesti ben precisi e ben delimitati nel tempo e nello spazio, da analizzare sulla base di tre caratteristiche fondamentali: il rapporto con l'ambiente, l'organizzazione sociopolitica e le espressioni culturali. Gli esempi dovevano essere scelti in modo da rappresentare, nell'arco dei due anni, tutti questi tipi di società, ed essere distribuiti nel tempo e nello spazio in modo da coprire vari momenti della storia, "dalla comparsa dell'homo sapiens ai giorni nostri» e le varie zone del pianeta, allo scopo di introdurre gli studenti nell'intera dimensione del tempo storico e nella dimensione spaziale mondiale ${ }^{27}$.

La fase successiva, quella "sistematica" del curricolo di storia comprendeva due parti: la prima, della durata di tre anni, fino alla fine della scuola di base, in cui erano previsti una serie di intrecci interdisciplinari con le altre due materie, in maniera però più autonoma che nella fase precedente; e la seconda, che copriva il periodo dall'inizio del XVIII secolo ai nostri giorni, fino alla fine del biennio, che sarebbe stata trattata successivamente, quando la commissione si sarebbe occupata della scuola superiore. Intanto venne preparato dunque l'elenco dei contenuti essenziali delle tre materie per gli ultimi tre anni della scuola di base, che venne inserito nella bozza del decreto di riforma della scuola

Cerini e Fiorin 2001, 110-111. In questo volume è pubblicato il testo finale del decreto, composto di due parti: il "Regolamento, recante norme in materia di curricoli della scuola di base, ai sensi dell'articolo 8 del decreto del presidente della Repubblica 8 marzo 1999, n. 275", e gli "Indirizzi per l'attuazione del curricolo". 
di base, presentato alla stampa il 7 febbraio $^{23}$. Per la storia erano indicati i seguenti contenuti:

\section{Quinto anno}

Il processo di ominazione e il popolamento della Terra

Le società di caccia e raccolta nel Paleolitico

La rivoluzione neolitica nel mondo: nomadi e sedentari

Le prime società urbane (Mesopotamia, Egitto, valle dell'Indo, Cina, America)

Sesto anno

La civiltà greca

La civiltà romana

Il cristianesimo

Migrazioni di nomadi, crisi e ristrutturazione degli imperi eurasiatici

L'espansione araba

L'Europa medievale

L'Impero mongolo

Africa subsahariana: la migrazione bantu; lo sviluppo degli stati

I Maya. L'Impero azteco. L'Impero inca

La colonizzazione dell'Oceania

\section{Settimo anno}

Lo stato moderno in Europa

La formazione degli stati regionali italiani

Umanesimo e Rinascimento

Riforma e Controriforma in Europa

L'espansione europea nel mondo: l'avvio del processo di globalizzazione

\footnotetext{
Il testo completo è stato pubblicato nell'inserto "Scuola" del quotidiano Il Sole 24 Ore (III, 4, 23 febbraio-8 marzo 2001).
} 


\section{L'Impero ottomano}

India, Cina e Giappone tra XVI e XVIII secolo

Nelle indicazioni metodologiche si precisava che questi contenuti avevano la funzione di punti di riferimento per costruire uno scenario della storia dell'umanità senza soluzione di continuità temporale e senza lacune spaziali, e se ne indicavano i seguenti fili conduttori: «il rapporto fra gli esseri umani e l'ambiente, l'organizzazione politico-sociale, il sistema degli scambi, le dinamiche di occupazione dei territori, le espressioni culturali» (Cerini e Fiorin 2001, 111). Nel generale scenario mondiale così costruito dovevano essere inseriti i vari approfondimenti tematici, fra cui quelli particolarmente significativi "per la costruzione del patrimonio culturale del cittadino italiano ed europeo» (ibid.), come la Grecia e la Roma classiche e la formazione del sistema degli Stati europei.

Per quanto riguarda i rapporti fra storia, geografia e scienze sociali erano previsti molti punti elementi di convergenza, su cui basare importanti sinergie nel contesto della programmazione. Ad esempio nel quinto anno, in parallelo con lo studio in storia del popolamento della Terra durante il Paleolitico si aveva in geografia lo studio della distribuzione attuale della popolazione sul pianeta e nelle scienze sociali lo studio delle dinamiche demografiche; sempre nello stesso anno, in parallelo con lo studio in storia delle prime società urbane si aveva in geografia lo studio del loro ambiente contemporaneo. Nel sesto anno lo studio delle origini del cristianesimo e dell'islam in storia si accompagnava allo studio dei fenomeni religiosi nelle scienze sociali. Nel settimo anno sia la geografia che le scienze sociali affrontavano questioni legate alle relazioni internazionali, dalla gestione degli aiuti umanitari, allo sviluppo sostenibile e alla Dichiarazione universale dei diritti umani. Geografia e scienze sociali erano fra l'altro i contesti in cui veniva sviluppato lo studio del tempo presente, in costante dialettica con il passato studiato in storia. Il sottogruppo di lavoro prefigurò infine anche la struttura 
dell'insegnamento della storia nel triennio, che sarebbe stato caratterizzato da una serie di approfondimenti tematici, cronologicamente ordinati, per avvicinare gli studenti alle procedure della ricerca, e queste indicazioni vennero già inserite nel testo definitivo del decreto:

Come meglio verrà precisato nelle indicazioni curricolari per la quota nazionale della secondaria, nella fase finale del curricolo (gli ultimi tre anni della scuola secondaria) gli studenti riprenderanno una seconda volta l'intera storia generale e cronologica come quadro di riferimento all'interno del quale si potranno sviluppare temi specifici che verranno trattati con particolare attenzione alle fonti e al dibattito storiografico, in modo da sviluppare l'approccio critico alla disciplina (ivi, 136).

Appena venne reso noto il curricolo di storia si scatenarono le polemiche. Due le critiche principali: la prima riguardava proprio l'elemento qualificante, cioè la dimensione mondiale, mentre la seconda contestava la continuità del curricolo di storia fino alla fine dell'obbligo scolastico, con l'intento di snaturare il carattere di transizione del biennio e di salvaguardare la tradizionale compattezza quinquennale della scuola secondaria, negando così uno dei fondamenti della riforma. Due furono in particolare i testi più significativi di queste critiche. In primo luogo il manifesto, significativamente intitolato Insegnamento della storia e identità europea, fra cui Paolo Prodi, Pasquale Villani, Luciano Canfora, Franco Della Peruta, Giuliano Procacci, Aurelio Lepre, Francesco Traniello, Francesco Perfetti, Giuseppe Galasso e Rosario Villari ${ }^{29}$, i quali reclamavano una totale «ridefinizione» del programma:

in modo da evitare il rischio che la pur necessaria visione mondiale dello sviluppo storico pregiudichi la piena valorizzazione dell'identità cultu-

Rosario Villari, in un’intervista, aveva già dichiarato che "uno studio della storia coincide con l'esigenza di conoscere approfonditamente l'identità della propria civiltà, della nazione e della comunità civile alle quali si appartiene» (Conti, P. 2001. "Villari: caro ministro, ecco perché la tua riforma è sbagliata." Il Corriere della Sera, 13 febbraio). 
rale italiana ed europea, e appiattisca le diversità di valori e di conquiste civili ${ }^{30}$.

Alcuni dei firmatari di questo manifesto, Girolamo Arnaldi, Massimo Firpo, Nicola Tranfaglia e Giovanni Vitolo, insieme a Piero Bevilacqua e a Cosimo Damiano Fonseca, redassero poi un Progetto per l'insegnamento della storia nella scuola di base e in quella superiore (Lineatempo 1, 106-112). In questo testo, come già nel manifesto, risalta la funzione identitaria che si voleva dare all'insegnamento della storia. Nel primo paragrafo, intitolato "Finalità e ruolo della conoscenza storica", si legge infatti:

Lo studio della storia [...] svolge una funzione centrale nel processo formativo fin dagli anni dell'infanzia sia perché le categorie storiche sono una delle chiavi fondamentali di lettura di tutta la realtà sia perché essa ha un ruolo fondamentale nella strutturazione della memoria e della coscienza nazionale e di gruppo (ivi, 106).

L'importanza della dimensione nazionale nell'insegnamento della storia risalta anche alla fine di questo paragrafo, dove si legge un'enfatica e assai discutibile rivendicazione del primato dell'Italia, del tutto discutibile sul piano storiografico:

[Alla storia n.d.a.] va riconosciuto [...] il significato di vera e propria struttura portante della scuola, sia di base sia superiore, per quanto attiene ai suoi compiti di formazione generale, soprattutto in un paese come l'Italia, dove l'innestarsi del cristianesimo e della Chiesa sull'eredità del mondo antico ha garantito una continuità storica senza uguali nel mondo (ibid.).

Gli estensori del Progetto insistevano ancora sulla dimensione identitaria collettiva come centro e pietra di paragone della conoscenza storica. Nel secondo paragrafo, intitolato "L'insegnamento della storia”, essi ri-

\footnotetext{
30 Il testo del manifesto è stato pubblicato sul Corriere della Sera, 25 febbraio 2001 ("Trentatré storici scrivono a De Mauro: la storia si salva così) e ripubblicato su $\mathrm{Li}_{-}$ neatempo 1, 2001: 105.
} 
badivano infatti che «la necessaria apertura multiculturale può e deve avvenire solo muovendo dalla conoscenza della propria cultura» (ivi, 107) e che è necessario "fondare la conoscenza delle storie 'altre' anzitutto su quella della propria» (ibid.). E più avanti, nel paragrafo intitolato "Criteri di selezione dei contenuti", essi affermavano che:

velleitaria è $[\ldots]$ la pretesa di estendere lo studio ad una dimensione mondiale per far sì che la storia d'Europa e quella degli altri continenti siano posti sullo stesso piano (ivi, 108).

Nel Progetto, dunque, la conoscenza della storia extraeuropea diventava marginale rispetto a quella "propria". Sempre in questo paragrafo, dove peraltro la dimensione collettiva di riferimento non era più quella italiana ma diventava quella europea, gli estensori affermavano infatti:

è molto importante $[\ldots]$ che il bambino sia messo in grado di riconoscere le sue radici, la sua identità culturale e che conosca perciò il passato europeo: passato che del resto gli parla - se sarà messo in grado di ascoltarlo - da tutto il paesaggio umano che lo circonda (ibid.).

Acquisita questa coscienza storica - continuavano gli estensori - lo studente avrebbe potuto rivolgere la sua attenzione all'eventuale compagno di banco extraeuropeo:

Non si deve perciò proporre che si studi il mondo intero, in modo enciclopedico e in pillole [...] ma che si studi in modo selettivo, partendo dalla nuova realtà che circonda il bambino. Ad esempio invece che dedicare lo stesso numero di pagine ai bantu, agli aborigeni australiani e ai popoli del medio Oriente, sarà meglio soffermarsi di più sulla civiltà, la cultura, la religione, di quelle popolazioni di cui lo studente ha ormai conoscenza diretta nell'ambito della scuola e della città in cui vive. Quanto poi alle realtà più lontane dall'Europa, si lascerà alla sensibilità e alla professionalità degli autori dei testi scolastici e dei docenti l'individuazione del momento in cui è didatticamente più utile spostare su di esse l'attenzione dei ragazzi, allo scopo di coglierne sia i caratteri originali sia le analogie con i processi storici in atto in Occidente (ibid.).

Insomma, la storia, ad esempio, della Cina o dell'Africa subsahariana non sono importanti di per sé, come elementi indispensabili per capire 
la storia passata e presente non solo di tutto il mondo, ma anche di una sua qualsiasi sua parte, compresa l'Europa, sono invece utili solo nel caso in cui si abbia come vicino di banco un cinese o un senegalese, per poter dialogare con lui.

Dopo queste premesse programmatiche, gli estensori presentavano un programma di storia in due cicli, scanditi dalla cesura fra scuola di base e scuola secondaria. Per la prima, dopo i primi due anni dedicati agli «elementi predisciplinari» e ai "concetti basilari», iniziava un percorso quinquennale diacronico di storia, che terminava con lo studio del Novecento nell'ultima classe della scuola di base ${ }^{31}$. Nella scuola superiore era previsto lo studio della storia «in un nuovo ciclo quinquennale». Uno studio che, si precisava, "non ha assolutamente carattere di ripetitività, ma si configura come proposta di approfondimento, secondo un modello di apprendimento non già cumulativo, ma 'a spirale'» (ivi, 111).

Nel primo anno del biennio, secondo questo piano, si sarebbe studiato il periodo che va dalla preistoria all'apogeo dell'impero romano, e nel secondo quello che va dall'età tardoantica alla crisi del secolo XIV. Gli estensori affrontavano qui una delle questioni che erano state prese in esame anche durante i lavori della Commissione, e cioè il fatto che, con due cicli quinquennali siffatti, coloro che avessero lasciato la scuola alla fine dell'obbligo avrebbero avuto una preparazione storica monca, che dopo la fine del primo ciclo si sarebbe arrestata alla peste nera. La risposta che essi davano a questa obiezione era che il numero di coloro che lasciavano la scuola a 15 anni era in via di riduzione e si sarebbe ulteriormente ridotto nel tempo ("secondo le previsioni, dal 25 al 10\%»), e che quindi "non si può pregiudicare la formazione della stragrande maggioranza degli studenti che continuano gli studi» (ibid.).

L'articolazione cronologica dei contenuti era la seguente: classe III: dalla preistoria alla civiltà greca; classe IV: la civiltà romana e l'alto medioevo; classe V: dalla rinascita del secolo XI alla crisi del Seicento; classe VI: dall'età dell'assolutismo all'età dell'imperialismo; classe VII: il Novecento. 
Peraltro, un approfondimento della storia antica e medievale europea non appariva loro inopportuno, neanche per chi lasciasse precocemente la scuola, perché:

è necessario tener presente limportanza per un paese quale liltalia (ma questo vale anche nella prospettiva della costruzione dellidentità europea) di una conoscenza adeguata della storia greco-romana e di quella medievale, dato che sono quelle le età nelle quali sono nate la libertà di pensiero, la riflessione razionale, la coscienza individuale, la comunità politica dei cittadini, le grandi entità religiose che sono elementi fondamentali del mondo di oggi, e si è venuto formando l/Occidente europeo come spazio autonomo di civiltà rispetto al mondo greco-bizantino e a quello islamico (ibid.).

Comunque, concludevano gli estensori del Progetto, si poteva prevedere anche nei primi due anni, per chi lascia la scuola alla fine dell'obbligo,

un'opzione alternativa di Educazione storica e civica, finalizzata all'approfondimento in prospettiva storica di temi legati ai nostri tempi, quali le istituzioni parlamentari italiane ed europee, l'Unione europea, le istituzioni parlamentari (l'ONU, la FAO, l'UNESCO, ecc.) nonché all'acquisizione del lessico politologico e sociale (ibid.).

Nel frattempo, oltre alle critiche, arrivavano anche espressioni di sostegno al nuovo curricolo, soprattutto dal mondo della scuola, ma anche seppure in misura minore rispetto agli avversari - da parte degli storici, fra cui Angelo Massafra, Biagio Salvemini, Marcello Verga, Giuseppe Barone, Gaetano Quagliariello, Franco Cardini e Lutz Klinkhammer, che firmarono un documento a favore ${ }^{32}$. Alla fine De Mauro decise di non modificare la fisionomia del curricolo, e fece solo alcuni cambiamenti nella versione definitiva del testo, che fu poi inserito nel decreto. In particolare nella versione definitiva $i$ bantu, che avevano suscitato non solo l'attenzione degli autori del Progetto ma anche lo sdegno di

32 "Storia: un documento in difesa di De Mauro «Non riportiamo i programmi agli anni '80».” Il Corriere della Sera, 27 febbraio 2001. 
Giuseppe Galasso ${ }^{33}$ e Aurelio Lepre ${ }^{34}$, scomparvero per lasciare il posto a una formulazione più vaga, ma sostanzialmente simile alla precedente: "l'Africa subsahariana: migrazioni e sviluppo statale» (Cerini e Fiorin, 112. De Mauro inserì inoltre nel quinto anno due nuovi contenuti: «il Mediterraneo in età preclassica; culture e popolazioni di lingua semitica e indoeuropea nel Mediterraneo e in Europa» (ibid.).

Il decreto di riforma iniziò così il normale iter burocratico, attraverso il Consiglio di Stato e la Corte dei Conti, e l'aveva quasi concluso quando il 13 maggio 2001 ci furono le elezioni politiche che videro la vittoria della coalizione di centrodestra guidata da Silvio Berlusconi. Durante la campagna elettorale uno delle sue promesse era stata proprio la cancellazione della riforma della scuola iniziata dal centrosinistra ${ }^{35}$, e anzi Rocco Buttiglione, uno degli ideologhi della sua coalizione e per un momento indicato come futuro ministro proprio dell'istruzione, aveva dichiarato la sua particolare avversione al curricolo di storia e alla sua impostazione mondiale: "I giovani non devono studiare la storia universale, ma innanzitutto quella del loro paese. Inseguendo un

33 Galasso, G. 2001. "La storia non si fotocopia.” Il Mattino, 15 febbraio: «Sullo stesso piano sono disposte, ad esempio, l'Africa sub-sahariana, con le migrazioni bantu, e le più grandiose e decisive esperienze civili dell'umanità. Via! Che cosa significa questo sciocco enciclopedismo ed egalitarismo storico? Dove tutto ha lo stesso rilievo, niente ha davvero rilievo; e, con tutto il rispetto per tutti e con un senso profondo della pari dignità umana di tutti gli uomini che nella storia vivono e operano, l'Ellade e il Rinascimento, la «rivoluzione scientifica» moderna e le rivoluzioni americana e francese, la Cina e l'Islam sono una cosa e i Bantu e gli Aborigeni australiani sono un'altra cosa. Né i programmi scolastici varranno mai a mettere sullo stesso piano ciò che la storia ha messo su un piano diverso».

34 intervista ad Aurelio Lepre di Dario Fertilio: Fertilio, D. 2001. "Macché destra e sinistra, sono i programmi scolastici a indignare noi storici." Il Corriere della Sera, 26 febbraio: "È un certo confuso mondialismo [...] che trascura la storia europea e italiana mettendo sullo stesso piano l'Europa medievale e l'impero mongolo, oppure l'Africa sub-sahariana con le sue migrazioni bantù. Ed è proprio questo che ci indigna: l'idea che tutti gli argomenti, in fondo, siano da considerarsi uguali».

35 "Berlusconi: se vincerò abrogherò la riforma." La Gazzetta del Mezzogiorno, 8 febbraio 2001. 
astratto cosmopolitismo, si annoiano. Devono capire la cultura in cui sono nati $»^{36}$. E così il nuovo ministro dell'Istruzione, dell'Università e della Ricerca (questa la nuova denominazione del ministero), Letizia Moratti, ritirò il decreto dalla Corte dei Conti prima che questa potesse esprimere il suo parere, annullando così tutto il lavoro fatto fino ad allora, e, sciolta la Commissione De Mauro, nominò un nuovo gruppo di lavoro (i cui componenti non vennero resi noti) per scrivere una nuova riforma della scuola.

Nel fallimento di questo tentativo di introdurre una visione mondiale della storia nella scuola italiana non entrarono soltanto motivazioni politiche e una visione identitaria della storia, ma anche una caratteristica propria della cultura storiografica italiana, come mise in luce Giuseppe Ricuperati. Egli, pur riconoscendo che il curricolo di storia della commissione De Mauro aveva rappresentato un'«interessante e dignitosa esperienza» (Ricuperati 2003, 771 ss.), rilevava che esso era stato percepito dagli storici che lo avversarono come «una minaccia per l'identità della disciplina» (ivi, 772), giacché «non solo manca una vera tradizione di World History nella ricerca italiana, ma è ancora del tutto recente una prospettiva storica «transnazionale» (ivi, n. 128).

\section{La riforma Moratti}

La riforma Moratti rappresentò un sostanziale ritorno al passato, con il ristabilimento della durata complessiva della scuola a 13 anni e la netta separazione fra un primo e un secondo ciclo, senza il biennio di raccordo $^{37}$. Il primo ciclo accorpava la scuola primaria (l'ex scuola elementare)

Intervista di Aldo Cazzullo a Rocco Buttiglione: "Buttiglione: così rivisiterò la storia. "Bisogna liberarla dalle incrostazioni marxiste».” La Stampa, 16 maggio 2001.

37 Legge 28 marzo 2003, n. 53: "Delega al Governo per la definizione delle norme generali sull'istruzione e dei livelli essenziali delle prestazioni in materia di istruzione e formazione professionale." Gazzetta Ufficiale 77, 2 aprile 2003. 
e la scuola secondaria di primo grado (l'ex scuola media), il che sanciva una tendenza sempre più diffusa nella scuola italiana, cioè la creazione degli istituti comprensivi, che mettevano insieme scuole elementari e medie sul piano amministrativo. Inoltre, nel primo ciclo venne introdotta la continuità del curricolo di storia fra l'ex scuola elementare e l'ex scuola media, al posto dei due programmi separati, che ripetevano due volte l'intero arco cronologico dalla preistoria all'età contemporanea nelle elementari e nelle medie. Veniva in tal modo recepito, anche se in un contesto diverso, un elemento della riforma De Mauro. L'impostazione generale della riforma Moratti corrispondeva a quella visione identitaria basata su cristianesimo, italianità ed europeismo, che era stata propugnata dagli estensori del Progetto. Infatti nel Profilo educativo, culturale e professionale dello studente alla fine del Primo Ciclo di istruzione (6-14 anni), che definiva il fine generale dell'insegnamento, si affermava che lo studente

ha consapevolezza, sia pure in modo introduttivo, delle radici storicogiuridiche, linguistico-letterarie e artistiche che ci legano al mondo classico e giudaico-cristiano, e dell'identità spirituale e materiale dell'Italia e dell'Europa; colloca, in questo contesto, la riflessione sulla dimensione religiosa dell'esperienza umana e l'insegnamento della religione cattolica...; sa orientarsi nello spazio e nel tempo, operando confronti costruttivi fra realtà geografiche e storiche diverse, per comprendere, da un lato, le caratteristiche specifiche della civiltà europea e, dall'altro, le somiglianze e le differenze tra la nostra e le altre civiltà del mondo; sa collocare, in questo quadro, i tratti spaziali, temporali e culturali dell'identità nazionale e delle identità regionali e comunali di appartenenza ${ }^{38}$.

Per quanto riguarda in particolare l'insegnamento della storia, non più collegata a geografia e scienze sociali, se ne prevedeva l'inizio con un'impostazione cronologica già nel corso del secondo e terzo anno

\footnotetext{
38 Decreto legislativo 19 febbraio 2004, n. 59: "Definizione delle norme generali relative alla scuola dell'infanzia e al primo ciclo dell'istruzione, a norma dell'articolo 1 della legge 28 marzo 2003, n. 53." Gazzetta Ufficiale 51, 2 marzo 2004, Supplemento ordinario 31, 109.
} 
della scuola primaria, con la "comparsa dell'uomo», «la rivoluzione neolitica» e «il passaggio dall'uomo preistorico all'uomo storico nelle civiltà antiche» ${ }^{39}$. Nel successivo biennio era previsto lo studio delle "grandi civiltà dell'Antico Oriente (Mesopotamia, Egitto, India, Cina) fino alla dissoluzione dell'impero romano»*0. Per il primo biennio della scuola secondaria di primo grado era poi previsto lo studio della storia fino alla Rivoluzione francese, e quindi nell'ultimo anno tornava lo studio dell'intero Ottocento, a partire da Napoleone, col che si cancellava il decreto Berlinguer sull'insegnamento del Novecento. L'elenco delle conoscenze era tutto incentrato sull'Italia e sull'Europa, a parte le grandi civiltà antiche già citate e la nascita dell'Islam. La volontà di sottolineare l'identità europea risalta ad esempio a proposito del Medioevo, dove si legge: «la civiltà europea dopo il Mille e l'unificazione culturale e religiosa dell'Europa: le radici di una identità comune pur nella diversità dei diversi sistemi politici»»1 . Anche a proposito di momenti in cui è centrale lo scambio continentale, come l'inizio dell'età moderna, la formulazione spostava l'accento esclusivamente sull'Europa: «l'apertura dell'Europa ad un sistema mondiale di relazioni: la scoperta dell' "altro" e le sue conseguenze»". Perplessità suscitano i contenuti dell'ultimo anno: vi mancavano ad esempio concetti storiografici fondamentali come «rivoluzione industriale», "colonialismo» e "imperialismo", celati, questi ultimi due, dietro due definizioni anodine: «l'Europa ed il mondo degli ultimi decenni dell'Ottocento» e «la competizione tra Stati e le sue conseguenze» ${ }^{{ }^{3}}$. E stupisce che fra la Prima e la Seconda guerra mondiale venisse collocato un tema dal titolo «l'età delle masse e la

Ivi, 39.

40 Ivi, 50-51.

${ }_{41}$ Ivi, 81.

42 Ibid.

$43 \quad$ Ivi, 92. 
fine della centralità europea»" dove la copula metteva in relazione due fenomeni non collegabili e neppure contemporanei. Colpisce infine il costante richiamo alle dimensioni regionali e locali dell'Italia, tema caro in particolare alla Lega Nord, che faceva parte della coalizione di centrodestra. Ad esempio, fra le abilità disciplinari del primo biennio figurava lo «scoprire specifiche radici storiche medievali e moderne nella realtà locale e regionale» ${ }^{\star 5}$ e fra le conoscenze dell'ultimo anno «lo stato nazionale italiano e il rapporto con le realtà regionali», insieme al «significato di simboli quali la bandiera tricolore, gli stemmi regionali, l'inno nazionale»+6.

A differenza di quanto era accaduto per il curricolo De Mauro, questo curricolo di storia non suscitò alcuna polemica né fra gli opinionisti né fra gli storici, segno che tutto sommato andava incontro alla communis opinio.

Nell'ottobre 2005 la Moratti promulgò poi le Norme generali ${ }^{\natural 7}$ per il secondo ciclo, con allegate le Indicazioni nazionali per i piani di studio personalizzati per i licei ${ }^{\ddagger}$. Mancavano quelle per il «sistema dell'istruzione e formazione professionale», che era stato passato alla competenza delle Regioni. Per quanto riguarda il secondo ciclo, gli «obiettivi specifici di apprendimento" per la storia (uguali per tutti i licei, anche se con monte orario diverso) si caratterizzavano per l'assenza di indicazioni metodologiche specifiche (il che valeva del resto per tutte le materie). Divisi

\footnotetext{
$44 \quad$ Ibid.

45 Ivi, p. 81.

$46 \quad$ Ivi, p. 92.

47 Decreto Legislativo 17 ottobre 2005, n. 226: "Norme generali e livelli essenziali delle prestazioni relativi al secondo ciclo del sistema educativo di istruzione e formazione, a norma dell'articolo 2 della legge 28 marzo 2003, n. 53." Gazzetta Ufficiale 257, 4 novembre 2005, Supplemento ordinario 175/L.
}

48 Il nuovo sistema dei licei assorbiva gli istituti tecnici e veniva così a comprendere i licei artistico, classico, economico, linguistico, musicale e coreutico, scientifico, tecnologico e delle scienze umane. 
su due bienni e un anno finale, erano organizzati su due colonne, prive di intitolazione, ma che evidentemente contenevano l'una le conoscenze e l'altra le abilità disciplinari, secondo lo schema già adottato per il primo ciclo. L'elenco delle conoscenze, piuttosto ampio, mostrava anche in questo caso l'assoluta prevalenza dell'Italia e dell'Europa, il che corrispondeva agli obiettivi di tutta la scuola, quali venivano dichiarati all'art. 1 \& 3 delle Norme generali: "la formazione intellettuale, spirituale e morale, anche ispirata ai principi della Costituzione, lo sviluppo della coscienza storica e di appartenenza alla comunità locale, alla collettività nazionale ed alla civiltà europea»*9. $\mathrm{E}$ in effetti, fra le conoscenze, $\mathrm{i}$ riferimenti al resto del mondo erano rarissimi e generici, similmente a quanto si è visto per il primo ciclo: «L'impero romano e le altre egemonie del mondo antico (Persia, India e Cina)»; «l'Islam e la formazione del califfato»; «la geografia del Nuovo Mondo e la creazione dei primi Imperi coloniali»; «Onu, decolonizzazione e neocolonialismo»; «lo sviluppo dell'Occidente, i paesi di nuova industrializzazione e il Sud del mondo»; «l'attuale quadro geopolitico mondiale»50. Da notare che la storia dell'ultimo anno non comprendeva tutto l'Ottocento, come nel primo ciclo, ma solo la seconda metà di questo secolo, a partire dalla seconda rivoluzione industriale e dall'unificazione italiana ${ }^{51}$.

\section{La riforma Fioroni}

Questa riforma del secondo ciclo sarebbe dovuta entrare in vigore nell'anno scolastico 2007-2008, ma, come era già avvenuto per la riforma De Mauro, anche questa rimase vittima del mutare degli eventi

\footnotetext{
49 Decreto Legislativo 17 ottobre 2005, 3.

50 Ivi, 73, 82, 90-91.

${ }^{51}$ Per una disamina più ampia dei contenuti di questo curricolo di storia si veda Valleri 2005, 3-34.
} 
politici. Le elezioni politiche del 9 e 10 aprile 2006 videro infatti la vittoria della coalizione di centrosinistra, e il nuovo ministro Giuseppe Fioroni bloccò la riforma del secondo ciclo $^{52}$ e cancellò quella della scuola dell'infanzia e del primo ciclo, con la pubblicazione di nuove Indicazioni per il curricolo ${ }^{53}$. Uno dei temi di fondo della riforma Fioroni fu il ruolo della scuola in un contesto multiculturale, una realtà che ormai si riconosceva anche in Italia. Nella parte che tratta le finalità generali dell'insegnamento si legge infatti:

Il sistema educativo deve formare cittadini in grado di partecipare consapevolmente alla costruzione di collettività più ampie e composite, siano esse quella nazionale, quella europea, quella mondiale. Non dobbiamo dimenticare che fino a tempi assai recenti la scuola ha avuto il compito di formare cittadini nazionali attraverso una cultura omogenea. Oggi, invece, può porsi il compito più ampio di educare alla convivenza proprio attraverso la valorizzazione delle diverse identità e radici culturali di ogni studente ${ }^{54}$.

Si trattava dunque di un netto cambiamento di rotta rispetto all'impostazione etnocentrica e identitaria della riforma Moratti, implicitamente criticata là dove si parlava del compito affidato alla scuola «fino a tempi assai recenti», e in questo cambiamento il ruolo principale venne naturalmente affidato alla storia, il cui curricolo venne redatto da un ristretto gruppo di storici italiani. Nell'ampia introduzione a questo curricolo si riprendeva appunto il tema del multiculturalismo e si metteva in guardia contro il pericolo che un'impostazione identitaria ed etnocentrica italiana o europea potesse generare reazioni e conflitti:

Nei tempi più recenti [...] il passato e in particolare i temi della memoria, dell'identità e delle radici hanno fortemente caratterizzato il di-

\footnotetext{
52 Reggio, M. 2006. “Scuola, stop alla riforma dei licei.” La Repubblica, 1 giugno.

53 Ministero della Pubblica Istruzione, Decreto 31 luglio 2007: "Indicazioni per la scuola d'infanzia e del primo ciclo di istruzione. Indicazioni per il curricolo." Gazzetta Ufficiale 228, 1 ottobre 2007, Supplemento ordinario 198.
}

54 Ivi, 11. 
scorso pubblico e dei media sulla storia. In tale contesto, la padronanza degli strumenti critici permette di evitare che la storia venga usata strumentalmente e in modo improprio. Inoltre, la formazione di una società multietnica e multiculturale ha portato con sé la tendenza a trasformare la storia da disciplina di studio a luogo di rappresentanza delle diverse identità, con il rischio di comprometterne il carattere scientifico e, conseguentemente, di diminuire la stessa efficacia formativa del curricolo. Per tale motivo, è opportuno sottolineare come proprio la storia offra una base solida per ragionare sulle diversità dei gruppi umani che hanno popolato il pianeta, a partire dall'unità del genere umano. In questo ambito acquisisce un rilievo centrale la trattazione di argomenti la cui conoscenza è imprescindibile per tutti gli alunni, da qualsiasi luogo provengano: dal Neolitico alla Rivoluzione industriale, dalla storia dell'ambiente a quella dei processi di globalizzazione ${ }^{55}$.

Nelle ultime righe di questa citazione si evoca una visione mondiale della storia come il contesto più adatto a evitare conflitti identitari. Ma gli estensori insistevano sul fatto che anche la storia italiana ed europea presentano elementi che consentono di «avviare il dialogo fra le diverse componenti di una società multiculturale e multietnica» e di «aprire la scuola a un confronto sereno ed educativo sui temi delle identità e delle differenze» ${ }^{56}$. Infatti, di questa storia venivano messi in luce soprattutto il «continuo rimescolamento di genti e di culture» attraverso $\mathrm{i}$ "profondi intrecci [...] fra le genti del Mediterraneo e le popolazioni dei continenti europei, asiatici e africani» ${ }^{57}$.

Altri riferimenti alla storia mondiale non ce n'erano, a parte una fuggevole citazione dell'India e della Cina come aree economiche e culturali che, al pari dell'Europa, «hanno caratterizzato la dinamica [...] del mondo moderno e contemporaneo ${ }^{58}$. I contenuti non erano elencati anno per anno, come nel curricolo De Mauro e in quello Moratti, ma inseriti

Ivi, 49-50.

Ivi, 50.

Ibid.

Ibid. 
all'interno dell'introduzione: oltre a quelli già citati, si trovano «le città, medievali e moderne» e il gruppo di quelli che sono definiti «i processi culturali che hanno caratterizzato vicende che sono state prima europee e poi mondiali: dalla nascita e dalla diffusione del Cristianesimo all'esordio dell'Umanesimo e del Rinascimento, alla Rivoluzione scientifica e all'Illuminismo» ${ }^{59}$. Seguiva la «formazione degli stati ottocenteschi e, sicuramente con maggiore attenzione, quella dello Stato Italiano ${ }^{60}$. Più dettagliato era l'elenco per il Novecento: "le guerre mondiali, il fascismo, il comunismo, la liberaldemocrazia, la decolonizzazione e le complesse vicende - economiche, sociali, politiche e culturali - che caratterizzano il mondo attuale; la formazione dell'Unione Europea; la nascita e le vicende della Repubblica italiana» ${ }^{61}$. C'era dunque una discrasia fra questo insieme di contenuti fondamentalmente eurocentrici e i richiami alla necessità di una dimensione mondiale, ribadita anche fra gli «obiettivi di apprendimento» da conseguire alla fine della classe terza della scuola secondaria di primo grado, dove era inserita la conoscenza dei "processi fondamentali della storia mondiale, dalla rivoluzione neolitica alla rivoluzione industriale alla globalizzazione». In questo il curricolo Fioroni era ben diverso da quello De Mauro, che aveva costruito un dettagliato quadro di riferimento di storia dell'umanità. Del curricolo De Mauro questo curricolo riprendeva invece la successione delle fasi didattiche, anche se con alcune differenze. A quella che era stata la fase iniziale della "grammatica», incentrata sui quadri di società, corrispondeva ora una prima fase di «formazione dei concetti di base del ragionamento storico», cioè "famiglia, gruppo, regole, agri-

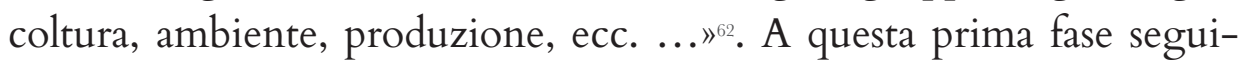

\footnotetext{
$59 \quad$ Ibid.

60 Ibid.

61 Ibid.

62 Ivi, 51.
} 
va quella della «conoscenza sistematica e diacronica»" da sviluppare a partire dall'ultimo biennio della scuola fino alla fine della scuola secondaria di primo grado. La cesura cronologica fra i due cicli scolastici era rappresentata dalla caduta dell'impero romano d'occidente, come nella riforma Moratti, ma a differenza di quella l'ultimo anno della scuola secondaria di primo grado era interamente riservato al Novecento. Va infine notato che questo curricolo di storia era collegato a quello di geografia nel formare l'area storico-geografica, ma che, a differenza che nel curricolo De Mauro, gli studi sociali (si era tornati alla definizione dei programmi per elementari del 1985 , al posto di scienze sociali) non avevano un curricolo autonomo, bensì ne era previsto lo sviluppo all'interno della storia e della geografia. Il ruolo di questa materia, sostanzialmente educazione alla cittadinanza, era in quegli anni oggetto di un ampio dibattito, che oscillava fra la sua totale autonomizzazione con proprio orario curricolare e la tradizionale incorporazione in materie affini, in primo luogo la storia. Il curricolo Fioroni sceglieva chiaramente questa seconda strada.

Queste Indicazioni entrarono subito in vigore: ne venne prevista un sperimentazione biennale, dopo la quale si sarebbe provveduto alla stesura definitiva ${ }^{64}$. Non ci fu invece tempo per mettere mano alla riforma del secondo ciclo. Infatti, nel 2008 la legislatura si chiuse prematuramente e le elezioni politiche del 13 e 14 aprile videro di nuovo la vittoria della coalizione di centrodestra.

\section{La riforma Gelmini}

Il nuovo ministro Maria Stella Gelmini lasciò in vigore le Indicazioni per il primo ciclo di Fioroni e riprese la riforma del secondo ciclo,

\footnotetext{
63 Ibid.

${ }_{64}$ Ivi, artt. 2 e 3.
} 
modificando peraltro in più punti sia l'architettura che i contenuti di quella redatta dalla Moratti. A livello di architettura della scuola superiore licei e istituti tecnici vennero di nuovo separati, e i curricoli degli istituti professionali tornarono di nuovo di competenza del ministero ${ }^{65}$. Per quanto riguarda la storia ${ }^{66}$, vanno poi rilevate due differenze di fondo: in primo luogo il ripristino della scansione che assegnava all'ultimo anno lo studio del solo Novecento; in secondo luogo, l'accorpamento di storia e geografia nel biennio dei licei, con un voto unico: una scelta dettata dalla riduzione del monte orario complessivo delle due materie (dalle 4 ore dell'ex ginnasio a 3 nel nuovo sistema). Nell'introduzione al curricolo di storia si giustificava l'accorpamento con questa proposta didattica:

Il punto di partenza sarà la sottolineatura della dimensione temporale di ogni evento e la capacità di collocarlo nella giusta successione cronologica, in quanto insegnare storia è proporre lo svolgimento di eventi correlati fra loro secondo il tempo. D'altro canto non va trascurata la seconda dimensione della storia, cioè lo spazio. La storia comporta infatti una dimensione geografica; e la geografia umana, a sua volta, necessita

${ }_{65}$ Il piano della riforma è contenuto nei tre seguenti decreti del presidente della Repubblica pubblicati in Gazzetta Ufficiale 137, 15 giugno 2010, Supplemento ordinario 128/L: Decreto del presidente della Repubblica del 15 marzo 2010, 87: "Regolamento recante norme per il riordino degli istituti professionali, a norma dell'articolo 64, comma 4, del decreto-legge 25 giugno 2008, n. 112, convertito, con modificazioni, dalla legge 6 agosto 2008, n. 133"; Decreto del presidente della Repubblica del 15 marzo 2010, 88: "Regolamento recante norme per il riordino degli istituti tecnici, a norma dell'articolo 64, comma 4, del decreto-legge 25 giugno 2008, n. 112, convertito, con modificazioni, dalla legge 6 agosto 2008, n. 133"; Decreto del presidente della Repubblica del 15 marzo 2010, 89: "Regolamento recante revisione dell'assetto ordinamentale, organizzativo e didattico dei licei, a norma dell'articolo 64, comma 4, del decreto-legge 25 giugno 2008, n. 112, convertito, con modificazioni, dalla legge 6 agosto 2008 , n. 133 ".

${ }_{66}$ Per un commento a questi programmi si veda il forum a cura di Cajani (2010), con interventi di C. Grazioli, M. Tarantini, G. Sergi, W. Panciera, T. Detti e G. De Vecchis. 
di coordinate temporali. Le due dimensioni spazio-temporali devono far parte integrante dell'apprendimento della disciplina ${ }^{67}$.

Il problema non si poneva invece nel biennio degli istituti tecnici e professionali, perché lì l'insegnamento della geografia venne abolito. Tornando all'introduzione al curricolo di storia, l'apertura poneva chiaramente l'accento sull'Italia e sull'Europa:

Al termine del percorso liceale lo studente conosce i principali eventi e le trasformazioni di lungo periodo della storia dell'Europa e dell'Italia, dall'antichità ai giorni nostri, nel quadro della storia globale del mon$\mathrm{do}^{68}$.

C'era anche un riferimento alla storia extraeuropea, tipicamente sporadico:

È utile ed auspicabile rivolgere l'attenzione alle civiltà diverse da quella occidentale per tutto l'arco del percorso, dedicando opportuno spazio, per fare qualche esempio, alla civiltà indiana al tempo delle conquiste di Alessandro Magno; alla civiltà cinese al tempo dell'impero romano; alle culture americane precolombiane; ai paesi extraeuropei conquistati dal colonialismo europeo tra Sette e Ottocento, per arrivare alla conoscenza del quadro complessivo delle relazioni tra le diverse civiltà nel Novecento $^{69}$.

Si trattava di qualcosa in più di ciò che contenevano le Indicazioni Moratti, ma certo l'impostazione eurocentrica non ne veniva minimamente scalfita: del resto del mondo si parla infatti solo quando l'Europa entra

Ministero dell'Istruzione, dell'Università e della Ricerca, Decreto 7 ottobre 2010, n. 211: "Schema di regolamento recante «Indicazioni nazionali riguardanti gli obiettivi specifici di apprendimento concernenti le attività e gli insegnamenti compresi nei piani degli studi previsti per i percorsi liceali di cui all'articolo 10, comma 3, del decreto del presidente della Repubblica 15 marzo 2010, 89, in relazione all'articolo 2, commi 1 e 3, del medesimo regolamento." Gazzetta Ufficiale 291, 14 dicembre 2010, Supplemento ordinario 275/L, 205.

68 Ivi, pp. 204-205.

$69 \quad$ Ivi, p. 205. 
in contatto con esso. E non a caso gli irrinunciabili "nuclei tematici", e i "temi cruciali" che formavano l'elenco dei contenuti (sotto la rubrica "Obiettivi specifici di apprendimento") erano tutti di storia europea, tranne qualche modesta, inevitabile eccezione per la storia più recente, dove non è proprio possibile ignorare Cina e India.

La scansione fra primo e secondo biennio era posta all'XI secolo. L'elenco dei contenuti era complessivamente molto più ampio di quello dei programmi precedentemente in vigore per i licei classico e scientifico (che nel momento in cui vennero aboliti risultarono essere stati i programmi più longevi di tutti, giacché l'ultima revisione risaliva al 1960). La storia antica era sintetizzata in soli 5 nuclei tematici: «le principali civiltà dell'Antico vicino Oriente», «la civiltà giudaica», «la civiltà greca», «la civiltà romana» e «l'avvento del Cristianesimo» ${ }^{70}$, con l'incomprensibile assenza della preistoria, presente invece già nei programmi precedenti e anche nelle Indicazioni Moratti. Al Medioevo erano dedicati 11 nuclei tematici, e altri 12 alla storia moderna (fino alla fine dell'Ottocento), e ovviamente molto dettagliato era il programma del Novecento, con particolare attenzione al secondo Novecento, di cui veniva presentato un quadro convincente:

Il quadro storico del secondo Novecento dovrà costruirsi attorno a tre linee fondamentali: 1) dalla "guerra fredda" alle svolte di fine Novecento: l'ONU, la questione tedesca, i due blocchi, l'età di Kruscev e Kennedy, il crollo del sistema sovietico, il processo di formazione dell'Unione Europea, i processi di globalizzazione, la rivoluzione informatica e le nuove conflittualità del mondo globale;2) decolonizzazione e lotta per lo sviluppo in Asia, Africa e America latina: la nascita dello stato d'Israele e la questione palestinese, il movimento dei non-allineati, la rinascita della Cina e dell'India come potenze mondiali; 3) la storia d'Italia nel secondo dopoguerra: la ricostruzione, il boom economico, le riforme degli anni Sessanta e Settanta, il terrorismo, Tangentopoli e la crisi del sistema politico all'inizio degli anni $90^{71}$.

Ivi, p. 206.

Ivi, p. 207. 
Nell'introduzione veniva anche indicato il modo di inserire l'educazione alla cittadinanza all'interno dell'insegnamento della storia, in linea con quanto si è già visto nel curricolo Fioroni:

uno spazio adeguato dovrà essere riservato al tema della cittadinanza e della Costituzione repubblicana, in modo che, al termine del quinquennio liceale, lo studente conosca bene i fondamenti del nostro ordinamento costituzionale, quali esplicitazioni valoriali delle esperienze storicamente rilevanti del nostro popolo, anche in rapporto e confronto con altri documenti fondamentali (solo per citare qualche esempio, dalla Magna Charta Libertatum alla Dichiarazione d'indipendenza degli Stati Uniti d'America, dalla Dichiarazione dei diritti dell'uomo e del cittadino alla Dichiarazione universale dei diritti umani), maturando altresì, anche in relazione con le attività svolte dalle istituzioni scolastiche, le necessarie competenze per una vita civile attiva e responsabile ${ }^{22}$.

A differenza delle Indicazioni Moratti, mancava in questo curricolo di storia per i licei una colonna delle abilità, accanto a quella delle conoscenze. Questa struttura riappariva invece nei curricoli di storia degli istituti tecnici e degli istituti professionali, che si distaccavano anche per altri aspetti da quelli dei licei e presentavano invece fra di loro molti elementi convergenti, anche grazie al fatto che gli istituti professionali erano ormai strutturati su un ciclo quinquennale. Nelle introduzioni generali ai rispettivi curricoli di storia risaltano due elementi: una funzionalizzazione dell'insegnamento della storia agli specifici contenuti tecnici e professionali e una sua forte dimensione valoriale. In quella per gli istituti tecnici si legge infatti:

Il docente di "Storia" concorre a far conseguire allo studente, al termine del percorso quinquennale, risultati di apprendimento che lo mettono in grado di: collocare le scoperte scientifiche e le innovazioni tecnologiche in una dimensione storico-culturale ed etica, nella consapevolezza della storicità del sapere; analizzare criticamente il contributo apportato dalla scienza e dalla tecnologia allo sviluppo dei saperi e dei valori, al cambiamento delle condizioni di vita e dei modi di fruizione culturale;

Ivi, p. 205. 
riconoscere l'interdipendenza tra fenomeni economici, sociali, istituzionali, culturali e la loro dimensione locale / globale; stabilire collegamenti tra le tradizioni culturali locali, nazionali ed internazionali sia in una prospettiva interculturale sia ai fini della mobilità di studio e di lavoro; essere consapevole del valore sociale della propria attività, partecipando attivamente alla vita civile e culturale a livello locale, nazionale e comunitario; valutare fatti ed orientare i propri comportamenti in base ad un sistema di valori coerenti con i principi della Costituzione e con le carte internazionali dei diritti umani; riconoscere gli aspetti geografici, ecologici, territoriali dell'ambiente naturale ed antropico, le connessioni con le strutture demografiche, economiche, sociali, culturali e le trasformazioni intervenute nel corso del tempo ${ }^{73}$.

Sostanzialmente simile era l'introduzione generale al curricolo di storia per gli istituti professionali, anche se più sintetica e con i valori costituzionali al primo posto $^{74}$. Per quanto riguarda poi le introduzioni specifiche per il primo biennio, va rilevato il forte riferimento alla contemporaneità anche nella trattazione della storia antica e medievale. Per entrambi gli istituti si indicava infatti l'obiettivo di «mettere in grado lo studente [...] di attribuire significato alle principali componenti storiche della contemporaneità confrontando aspetti e processi presenti con quelli del passato» ${ }^{75}$, e si invitava esplicitamente a privilegiare come strategia didattica il rapporto presente-passato-presente, un modello introdotto nei programmi del 1997 per gli istituti professionali ${ }^{76}$, e che

${ }_{73}$ Ministero dell'Istruzione, dell'Università e della Ricerca, Direttiva 15 luglio 2010: "Linee guida per il passaggio al nuovo ordinamento degli istituti tecnici a norma dell'articolo 8, comma 3, del decreto del presidente della Repubblica, 15 marzo 2010, n. 88. (Direttiva n. 57)." Gazzetta Ufficiale 222, 14 dicembre 2010, 44.

${ }^{74}$ Ministero dell'Istruzione, dell'Università e della Ricerca, Direttiva 15 luglio 2010: "Linee guida per il passaggio al nuovo ordinamento degli istituti professionali a norma dell'articolo 8, comma 6, del decreto del presidente della Repubblica, 15 marzo 2010, n. 87. (Direttiva n. 65)." Gazzetta Ufficiale 222, 14 dicembre 2010, 134.

75 Si vedano le note 62 e 63.

${ }^{76}$ Ministero della Pubblica Istruzione, Decreto Ministeriale 31 gennaio 1997: "Revisione dei programmi di storia per il triennio dei corsi di qualifica e per il biennio dei corsi post qualifica, e del programma di italiano per il terzo anno dei corsi di qualifica 
appare non privo di seri problemi sia sul piano scientifico che su quello didattico ${ }^{77}$.

Conoscenze e abilità erano identiche per entrambi gli istituti. L'elenco delle conoscenze era molto ridotto rispetto a quello dei licei, di cui ne riprendevano solo alcune, aggiungendovi però la preistoria ("la diffusione della specie umana sul pianeta»). Venivano poi aggiunti generici riferimenti alla storia economica e sociale, alla storia delle tecniche e del lavoro, al lessico di base della storiografia e alla Costituzione italiana. Nella colonna delle abilità risaltavano quelle cronologiche e geografiche, il confronto fra le interpretazioni e l'uso delle fonti, e il riconoscimento delle origini storiche delle attuali istituzioni politiche, economiche e religiose.

\section{La riforma Profumo}

L'ultimo atto di questo lungo processo di riforma è stato compiuto dal successore della Gelmini, Francesco Profumo, che è divenuto nel 2011 ministro del governo tecnico presieduto da Mario Monti e che ha completato la riforma degli istituti tecnici e professionali, pubblicando le Linee guida relative al secondo biennio e all'ultimo anno ${ }^{78}$, e ha revisionato le Indicazioni Fioroni, dopo la prevista sperimentazione.

dell'istruzione professionale." Gazzetta Ufficiale 36, 13 febbraio 1997, Supplemento ordinario 31 .

77 Per una critica di questo modello si veda Panciera e Zannini, 120-127.

${ }_{78}$ Ministero dell'Istruzione, dell'Università e della Ricerca, Direttiva 16 gennaio 2012: "Adozione delle Linee guida per il passaggio al nuovo ordinamento degli Istituti tecnici a norma dell'articolo 8, comma 3, del decreto del presidente della Repubblica 15 marzo 2010, n. 88 - Secondo biennio e quinto anno. (Direttiva n. 4).” e Ministero dell'Istruzione, dell'Università e della Ricerca, Direttiva 16 gennaio 2012: "Adozione delle Linee guida per il passaggio al nuovo ordinamento degli Istituti professionali a norma dell'articolo 8, comma 6, del decreto del presidente della Repubblica 15 marzo 
Per quanto riguarda l'insegnamento della storia negli istituti tecnici e professionali queste Linee guida sono in perfetta continuità con le precedenti quanto a subordinazione agli specifici obiettivi formatici dei due istituti. Nell'introduzione generale degli istituti tecnici questa funzione viene di nuovo chiaramente ribadita:

Le competenze storico-sociali [Storia] contribuiscono alla comprensione critica della dimensione teorico-culturale dei saperi e delle conoscenze proprie della scienza e della tecnologia attraverso lo sviluppo e l'approfondimento del rapporto fra le discipline delle Aree di indirizzo e la Storia e consentono allo studente, tra l'altro, di collocare le scoperte scientifiche e le innovazioni tecnologiche in una dimensione etica e storico-culturale; di riconoscere l'interdipendenza tra fenomeni economici, sociali, istituzionali, culturali e la loro dimensione locale/globale; di essere consapevole del valore sociale della propria attività, partecipando attivamente alla vita civile e culturale. [...] Approfondimenti di storie 'settoriali' (es.: storia dell'ambiente, storia economica e sociale, storia della scienza e della tecnologia) mettono in relazione le variabili privilegiate (es.: innovazioni tecnologiche) con altre variabili (es.: scoperte scientifiche, forme di organizzazione del lavoro, sistemi economici, modelli culturali) e con riferimento ad un contesto 'globale" ${ }^{79}$.

Molto simile anche l'analogo passaggio per gli istituti professionali ${ }^{80}$. L'elenco delle conoscenze, praticamente identico per entrambi gli istituti, è molto particolare: mentre nel biennio, come si è visto, ce n'era un certo numero in comune con i licei, questa volta la differenza è profonda, evidentemente a marcare più nettamente la diversa impostazione che si vuol dare all'insegnamento della storia. Non ci sono dunque eventi o fatti ben definiti, ma riferimenti a fenomeni molto generali, quali ad esempio, per il secondo biennio, "principali persistenza e processi di trasformazione tra il secolo XI e il secolo XIX in Italia, in Europa e nel mondo», «evoluzione dei sistemi politico-istituzionali

2010, n. 87 - Secondo biennio e quinto anno. (Direttiva n. 5)." Gazzetta Ufficiale 76, 30 marzo 2012, Supplemento ordinario 60.

79 Ministero dell'Istruzione, dell'Università e della Ricerca, Direttiva 16 gennaio 2012: "Adozione delle Linee guida per il passaggio al nuovo ordinamento degli Istituti tecnici.”, 13.

${ }^{80}$ Ministero dell'Istruzione, dell'Università e della Ricerca, Direttiva 16 gennaio 2012: "Adozione delle Linee guida per il passaggio al nuovo ordinamento degli Istituti professionali.", 310. 
ed economici, con riferimenti agli aspetti demografici, sociali e culturali», «innovazioni scientifiche e tecnologiche: fattori e contesti di riferimento", e, per il quinto anno, "principali persistenza e processi di trasformazione tra il secolo XIX e il secolo XXI in Italia, in Europa e nel mondo", "modelli culturali a confronto: conflitti, scambi e dialogo interculturale» e "problematiche sociali ed etiche caratterizzanti l'evoluzione dei settori produttivi e del mondo del lavoro ${ }^{81}$.

Per quanto riguarda la revisione delle Indicazioni Fioroni per il primo ciclo, il nuovo curricolo di storia ${ }^{82}$ presenta molte somiglianze e alcune differenze, in particolare per quanto riguarda l'aggiunta di una insistita riflessione sul patrimonio culturale italiano e sul significato particolare che la sua ricchezza dà all'insegnamento della storia in Italia. Nel primo paragrafo, significativamente intitolato "Il senso dell'insegnamento della storia" si legge infatti:

Nel nostro Paese la storia si manifesta alle nuove generazioni nella straordinaria sedimentazione di civiltà e società leggibile nelle città, piccole o grandi che siano, nei tanti segni conservati nel paesaggio, nelle migliaia di siti archeologici, nelle collezioni d'arte, negli archivi, nelle manifestazioni tradizionali che investono, insieme, lingua, musica, architettura, arti visive, manifattura, cultura alimentare e che entrano nella vita quotidiana. La Costituzione stessa, all'articolo 9, impegna tutti, e dunque in particolare la scuola, nel compito di tutelare questo patrimonio. Lo studio della storia, insieme alla memoria delle generazioni viventi, alla percezione del presente e alla visione del futuro, contribu-

Ministero dell'Istruzione, dell'Università e della Ricerca, Direttiva 16 gennaio 2012: "Adozione delle Linee guida per il passaggio al nuovo ordinamento degli Istituti tecnici.", 31-32.

82 Ministero dell'Istruzione, dell'Università e della Ricerca, Decreto 16 novembre 2012, n. 254: "Regolamento recante indicazioni nazionali per il curricolo della scuola dell'infanzia e del primo ciclo d'istruzione, a norma dell'articolo 1, comma 4, del decreto del presidente della Repubblica 20 marzo 2009, n. 89." Gazzetta Ufficiale 30, 5 febbraio 2013. I nomi dei funzionari ministeriali che l'hanno redatto e degli esperti coinvolti sono elencati a pagina 4. 
isce a formare la coscienza storica dei cittadini e li motiva al senso di responsabilità nei confronti del patrimonio e dei beni comunis3.

Il tema del patrimonio ritorna anche nell'ultimo paragrafo dell'introduzione, dove viene collegato all'educazione alla cittadinanza:

L'insegnamento e l'apprendimento della storia contribuiscono all'educazione al patrimonio culturale e alla cittadinanza attiva. I docenti si impegnano a far scoprire agli alunni il nesso tra le tracce e le conoscenze del passato, a far usare con metodo le fonti archeologiche, museali, iconiche, archivistiche, a far apprezzare il loro valore di beni culturali. In tal modo l'educazione al patrimonio culturale fornisce un contributo fondamentale alla cittadinanza attiva. In particolare, gli insegnanti metteranno in evidenza i rapporti tra istituzioni e società, le differenze di genere e di generazioni, le forme statuali, le istituzioni democratiche ${ }^{84}$.

Per il resto, questo curricolo riprende in pieno, parola per parola, le preoccupazioni della precedente versione in merito ai conflitti identitari possibili nelle società multiculturali. I due curricoli sono simili anche per quanto riguarda la scansione cronologica delle conoscenze nei vari cicli, con l'ultimo anno riservato al Novecento. Per l'impostazione del curricolo vengono indicati come «snodi periodizzanti della vicenda umana» alcuni elementi già presenti nel curricolo Fioroni: «il processo di ominazione, la rivoluzione neolitica, la rivoluzione industriale, i processi di mondializzazione e globalizzazione» ${ }^{85}$. Una periodizzazione potenzialmente mondiale, contraddetta però dall'elenco delle conoscenze, simili a quelle del curricolo Fioroni, anche se presentate in maniera sequenziale e più organica:

dal preistorico alle prime società del protostorico, dalle grandi civiltà antiche alla colonizzazione greca e al processo di unificazione del Mediterraneo, dalla costituzione dell'Impero Romano alla diffusione del

\footnotetext{
$83 \quad$ Ivi, 43.

84 Ivi, 45.

85 Ivi, 43.
} 
Cristianesimo, dalla progressiva strutturazione dei territori alla nascita di una società ricca per i diversi apporti di genti e di culture nel Medioevo; dall'Umanesimo e dal Rinascimento alle scoperte geografiche e all'espansione europea, dalla Riforma protestante alla costruzione degli stati moderni; dalla Rivoluzione scientifica all'Illuminismo e alla formazione di stati di diritto; dalla colonizzazione alla formazione degli stati nazionali, in particolare quello italiano, dall'industrializzazione al diffondersi della società di massa e all'emancipazione femminile; dai conflitti mondiali all'affermazione di dittature e all'espansione della democrazia, dai movimenti di resistenza alla formazione della Repubblica italiana, dalla decolonizzazione all'avvento della globalizzazione; dalle rivoluzioni scientifiche alla rivoluzione digitale ${ }^{86}$.

Un'impostazione quindi di nuovo tradizionalmente eurocentrica, senza neppure la menzione della Cina e dell'India che c'era nel curricolo Fioroni.

\section{Conclusioni}

Nel fare il bilancio di questo lungo e accidentato percorso di riforma la prima considerazione è che la forte spinta innovatrice della commissione De Mauro è stata sostanzialmente bloccata. Per quanto riguarda l'architettura del sistema, a parte il mantenimento del percorso scolastico di 13 anni, è rimasto intatto il blocco dei cinque anni della scuola superiore, e il progetto di un biennio di transizione è stato respinto. Una novità è però l'accorpamento dell'ex scuola elementare e dell'ex scuola media in un unico ciclo, il che per la storia ha portato alla continuità del curricolo nella scuola primaria: si hanno dunque ora due percorsi cronologici, e non più tre. Acquisita è invece la trattazione del Novecento nell'ultimo anno di ogni ciclo. Per quanto riguarda i contenuti dell'insegnamento della storia, la visione mondiale è stata respinta con

Ivi, 44. 
successo, e dopo quel primo tentativo nel 2001 è tornata incontrastata l'impostazione eurocentrica. Peraltro è fallito anche il principale avversario di questa visione mondiale, cioè il progetto etnocentrico e identitario della riforma Moratti: esplicitamente respinta nel primo ciclo, questa impostazione è del tutto ignorata nei programmi del secondo ciclo. Questo va e vieni può essere letto come lo specchio di un alternarsi di scelte politico-culturali nel ministero: a un'impostazione critica con De Mauro, in un governo di centrosinistra, è seguita un'impostazione fortemente connotata ideologicamente in senso identitario con il ministero Moratti, in un governo di centrodestra, impostazione a sua volta ribaltata dal successivo ministero Fioroni, di nuovo in un governo di centrosinistra. Con il ministero Gelmini, nonostante questo facesse parte come quello Moratti di una coalizione di centrodestra, la tensione ideologica si è smorzata e ne è scaturito un curricolo di storia sostanzialmente anodino sul piano identitario. Con il ministero Profumo si è continuato nella strada ormai tracciata da Fioroni, con un curricolo fortemente antietnocentrico per il primo ciclo, e dalla Gelmini, con il completamento dei curricoli per gli istituti tecnici e professionali. Visto il rilievo che il tema dell'identità etnocentrica ha avuto nelle polemiche contro il curricolo De Mauro e che continua ad avere nel dibattito politico e nell'opinione pubblica italiana, questo risultato, peraltro discordante fra i due cicli, è certamente da sottolineare. Tuttavia, la mancanza di un sostanziale cambiamento nei contenuti dell'insegnamento, che rimangono tradizionalmente eurocentrici, con appena qualche sfumatura diversa, vanifica almeno in buona parte anche le esplicite intenzioni antiidentitarie, proprio perché il punto di vista rimane eurocentrico e produce quindi una distorsione prospettica nella visione della storia dell'umanità, con il risultato di instillare implicitamente un'identità etnocentrica negli studenti. 


\section{Bibliografia}

Cajani, Luigi. 2003. "Italien und der Zweite Weltkrieg in den Schulgeschichtsbüchern. "In Erinnerungskulturen. Deutschland, Italien und Japan seit 1945, a cura di Lutz Klinkhammer, Christoph Cornelißen e Wolfgang Schwentker, 269-84, Frankfurt am Main: Fischer Taschenbuch Verlag.

- 2010. "I nuovi programmi di storia e geografia per i licei, gli istituti tecnici e gli istituti professionali." Mundus 5-6: 17-39.

Cerini, Giancarlo, e Italo Fiorin. 2001. I curricoli della scuola di base. Testi e commenti. Napoli: Tecnodid-Zanichelli.

Di Pietro, Gianni. 1991. Da strumento ideologico a disciplina formativa. I programmi di storia nell'Italia contemporanea. Milano: Edizioni scolastiche Bruno Mondadori.

Il biennio della scuola secondaria superiore. 1986. (Studi e documenti degli Annali della Pubblica Istruzione 38). Firenze: Le Monnier.

Le conoscenze fondamentali per l'apprendimento dei giovani nella scuola italiana nei prossimi decenni. I materiali della Commissione dei Saggi. 1997. (Studi e documenti degli Annali della Pubblica Istruzione 78). Firenze: Le Monnier.

Le conoscenze fondamentali per l'apprendimento dei giovani nella scuola italiana nei prossimi decenni. I materiali della Commissione dei Saggi, in "Studi e documenti degli Annali della Pubblica Istruzione», n. 78, Firenze, Le Monnier, 1997.

Lopes Don, Patricia. 2003. "Establishing World History as a Teaching Field: Comments from the Field." The History Teacher 36 (4, August): 505-525.

Nash, Gary B., Crabtree Charlotte A., e Ross E. Dunn. 1999. History on Trial. Culture Wars and the Teaching of the Past. New York: Alfred A. Knopp.

National Standards for History, Basic Edition. 1996. Los Angeles: National Center for History in the Schools.

Panciera, Walter e Andrea Zannini. 20133. Didattica della storia. Manuale per la formazione degli insegnanti. Firenze: Le Monnier.

Piani di studio della scuola secondaria superiore e programmi dei trienni. Le proposte della Commissione Brocca. 1992. (Studi e documenti degli Annali della Pubblica Istruzione 59/60). Firenze: Le Monnier.

Piani di studio della scuola secondaria superiore e programmi dei primi due anni. Le proposte della Commissione Brocca. 1991. (Studi e documenti degli Annali della Pubblica Istruzione 56). Firenze: Le Monnier.

Polacco, Fabrizio. 1999. "Storia e contesto storico." Annali della Pubblica Istruzione XLV (3-4): 141-143.

Progetto per l'insegnamento della storia nella scuola di base e in quella superiore. 2001. Lineatempo 1: 106-112. 
Ricuperati, Giuseppe. 2003. "A proposito di «Whose History?», e di uso pubblico della storia. Lo scontro sui piani di studio negli Stati Uniti (e in Italia)." Rivista storica italiana CXV: 733-779.

Valleri, Elvira. 2005. "«Lo spirito del mondo a cavallo». A margine dei nuovi programmi di storia.” Contemporanea 1: 3-34 (DOI: 10.1409/19274). 
